\title{
Bubbles in Kaluza-Klein theories with spacelike or timelike internal dimensions
}

\author{
A. Chamblin \\ Institute for Theoretical Physics, University of California, Santa Barbara, California 93106-4030 \\ R. Emparan \\ Department of Physics, University of California, Santa Barbara, California 93106
}

(Received 6 August 1996)

\begin{abstract}
Bubbles are pointlike regular solutions of the higher-dimensional Kaluza-Klein equations that appear as naked singularities in four dimensions. We analyze all such possible solutions in five-dimensional (5D) Kaluza-Klein theory that are static and spherically symmetric. We show that they can be obtained by taking unusual choices of the parameters in the dyonic black hole solutions, and find that regularity can only be achieved if their electric charge is zero. However, they can be neutral or possess magnetic charge. We study some of their properties, both in theories where the internal dimension is spacelike as well as timelike. Since bubbles do not have horizons, they have no entropy, nor do they emit any thermal radiation, but they are, in general, nonextremal objects. In the two-timing case, it is remarkable that nonsingular massless monopoles are possible, probably signaling a new pathology of these theories. These two-timing monopoles connect two asymptotically flat regions, and matter can flow from one region to the other. We also present a $C$-type solution that describes neutral bubbles in uniform acceleration, and we use it to construct an instanton that mediates the breaking of a cosmic string by forming bubbles at its ends. The rate for this process is also calculated. Finally, we argue that a similar solution can be constructed for magnetic bubbles, and that it can be used to describe a semiclassical instability of the two-timing vacuum against production of massless monopole pairs. [S0556-2821(97)01702-5]
\end{abstract}

PACS number(s): 04.50.+h, 04.20.Gz, 04.40.Nr, 04.70.Dy

\section{INTRODUCTION}

The solitonic solutions of the gravitational field equations have always been a focus of interest, much more in recent times after the recognition of their relevance towards a nonperturbative understanding of string theory [1]. We refer to solitons in a broad sense, as localized solutions of the classical equations of motion that are nonsingular or possibly have singularities hidden behind a horizon, the latter corresponding to black holes, or more generally, black branes. Certainly, to qualify as particle states one would also like the solutions to be stable. However, it has been proven for a wide range of solutions that nonextremal $p$-branes (with $p \geqslant 1$ ) are classically unstable [2]. Nonetheless, though unstable, many of these solutions are interesting on their own.

Most of the solitons that have been considered are of black hole type, i.e., objects with horizons and, correspondingly, an associated entropy - in some cases, though, the horizon area vanishes in the extremal limit, and singularities appear. However, there also exists an interesting class of solutions that, even if they look like naked singularities in four dimensions, their singularity can be resolved by going to a higher dimension. This resolution of singularities is purely classical, not involving any quantum smearing of the region surrounding the singularity, and it has been shown to smooth the inner singularity inside some black holes and black branes of interest [3]. If applied to resolve naked singularities, the outcome is a completely regular geometry for a pointlike object, though, as we will see, it is often an unstable one. Throughout the paper we will be working in the simplest theory in which these solutions can appear, namely five-dimensional Kaluza-Klein (KK) theory. Many of the features described here should also be present in more general theories with compact dimensions.

We will describe two ways in which a naked singularity can be regularized when "blown up" to reveal the higher dimensional structure of spacetime. In one situation, the internal space closes up smoothly-in the microscopic region representing the point particle-avoiding in this way the reaching of the singular origin. Geodesic completeness is preserved by leaving a nonsingular "hole" in space. In the other case, the pointlike particle turns out to be a tiny microscopic region bridging two asymptotically flat spacetimes through "chronology horizons."

Solutions of this kind have been known for some time as "bubbles," and this is the name we will adopt throughout this paper. An early realization of the importance of bubbles was found by Witten in [4], where it was argued that the Kaluza-Klein vacuum can decay by spontaneously forming bubbles that exponentially expand after their creation. In this paper, however, we will exclusively focus on nonexpanding bubbles. An interesting feature of all bubbles is that they do not have horizons, and therefore are zero-temperature objects with zero entropy.

Bubbles appear when the internal isometry along which the dimensional reduction is performed contains fixed points. The Gross-Perry-Sorkin (GPS) monopole $[5,6]$ is an example of a bubble in which the fixed point set consists of a single point and the bubble has zero size. In general, however, the fixed point set will be higher dimensional, and the bubble will have finite size. Several bubble solutions of fivedimensional (5D) Kaluza-Klein theory (with spacelike internal dimension) were already noticed in $[5,6]$, and also discussed in $[7,8]$. In this paper we aim at determining in a 
complete way all the possible static spherical bubble solutions of 5D Kaluza-Klein theory, clarify their relationship with the already thoroughly studied black hole solutions of the theory, and analyze bubble pair production and its consequences. Parallelly, we will study a much less explored issue, that of the solitonic objects in KK theories in which the internal dimension is timelike.

Theories with internal timelike dimensions have been a subject of interest for some time, and, in a rather modified way, have been recently revived in the context of string theory (" $F$ theory") [9]. However, not much is known about the classical vacuum solutions of two-timing theories. Recently, a full classification of the possible isometry groups of compact Lorentzian manifolds has been achieved [10], and this may be of relevance in determining the properties of compactifications in such theories. For the moment, however, we will restrict ourselves to the simplest case of a single internal timelike $S^{1}$.

The timelike character of an internal direction gives rise to several pathological features, the most conspicuous of which may be the fact that excitations of the internal dimensions have negative norm. Experimental lower bounds on possible violations of unitarity put a limit on the maximum radius of the internal timelike direction [11]. If one wants to preserve unitarity in a higher energy range, these degrees of freedom must be somehow frozen out or gauged away. An additional problem is that, as we will see in a moment, the coupling constant coming from the compactification modulus is imaginary. Oppositely charged objects then repel, and, by an old argument due to Dyson, one would expect the vacuum to be unstable against pair production of charged particles [12]. An instability of this kind was sought in [13], where it was noticed that the two-timing theory contains massless black holes, and solutions describing a pair of them accelerating apart can be found. However, due to peculiar thermal properties of these black holes, an instanton describing their pair creation could not be constructed. One of the aims of this paper is to show that this obstruction can be surmounted if instead of massless black holes one considers massless monopole bubbles, to be constructed below.

Our analysis of the bubble solutions in theories with internal time will be greatly simplified by noticing their relation with solutions with internal space: given a solution of the 5D Kaluza-Klein equations with spacelike internal dimension,

$$
d s^{2}=e^{-4 \phi / \sqrt{3}}\left(d x^{5}+2 A_{\mu} d x^{\mu}\right)^{2}+e^{2 \phi / \sqrt{3}} g_{\mu \nu} d x^{\mu} d x^{\nu},
$$

where $\phi$ is the scalar modulus ("dilaton"), $A_{\mu}$ the Maxwell four-potential, and $g_{\mu \nu}$ the effective four-dimensional metric $(\mu, \nu=0, \ldots, 3)$, a solution of the two-timing theory can be readily obtained by Wick rotating,

$$
x^{5} \rightarrow i x^{5}
$$

and, accordingly, in order to keep the solution real,

$$
A_{\mu} \rightarrow i A_{\mu} .
$$

The latter amounts to making $e^{2}$, the square of the electric charge, take on negative values, i.e., the coupling constant has been continued to an imaginary value and objects with equal sign charges attract each other. This method will allow us to easily generate bubble solutions of two-timing theories. However, even if formally similar, the two-timing bubbles will differ considerably in their properties from ordinary bubbles.

\section{BUBBLES IN 5D KALUZA-KLEIN WITH INTERNAL SPACELIKE DIRECTION}

We want to find all the topologically nontrivial regular solutions of 5D KK theory that appear as static, spherically symmetric, points acting as sources of the Maxwell field. Complete analyses of the static, spherically symmetric solutions of 5D KK theory have been given in different forms, see e.g., $[14,16]$, and also [15], but the focus has been generally on the black hole solutions. However, we will find that bubble solutions correspond to unconventional choices of the parameters in black hole solutions with both electric and magnetic charge. It is convenient to illustrate this first in the simplest example where the magnetic charges are zero.

It has been known for some time that, by taking the Schwarzschild solution and boosting it in the fifth direction, the entire family of electrically charged (magnetically neutral) KK black holes is generated. The five-dimensional metric in this case is

$$
\begin{gathered}
d s^{2}=\left(1+\frac{2 m \sinh ^{2} \delta_{Q}}{r}\right)\left(d x^{5}+2 A_{t} d t\right)^{2} \\
-\frac{1-2 m / r}{1+2 m \sinh ^{2} \delta_{Q} / r} d t^{2}+\frac{d r^{2}}{1-(2 m / r)}+r^{2} d \Omega_{2}^{2} \\
A_{t}=\frac{m \sinh 2 \delta_{Q}}{2 r\left(1+2 m \sinh ^{2} \delta_{Q} / r\right)}
\end{gathered}
$$

Here the electric charge $Q$ is a function of the boost parameter $\delta_{Q}$ :

$$
Q=\frac{m}{2} \sinh 2 \delta_{Q}=m \frac{v}{1-v^{2}},
$$

where $v$ is the boost velocity. By taking $0 \leqslant v<1$ we obtain, upon reduction, the spectrum of purely electric black holes. The limit $v \rightarrow 1$ (which requires $m \rightarrow 0$, keeping the charge and mass finite) describes an extremal singular solution.

What is less well known is what happens when we take $v>1$. Even if the boost parameter $\delta_{Q}$ in these solutions is complex, with imaginary part $i \pi / 2$, we still find real solutions. The four-dimensional reduced metric does not contain a horizon any more, but, rather, a naked singularity. However, we must analyze if this singularity is also present in the full five-dimensional geometry.

For all the values $1<v<\infty$ we have an electrically charged solution for which the internal circles close at 


$$
r=r_{B} \equiv 2 m \frac{v^{2}}{v^{2}-1}
$$

This closing of the internal space is the characteristic feature of bubbles with internal spacelike directions. However, for any finite $v>1$ the curvature is singular at $r_{B}$. This can be easily seen from the fact that, even if the length of the internal circles goes to zero at that point, their proper radius does not. Additionally, the $g_{t t}$ term diverges badly at the bubble.

The singularity disappears only for $v \rightarrow \infty$. Of course, this is equivalent to interchanging the roles of the internal and time coordinates in the Schwarzschild solution:

$$
d s^{2}=-d t^{2}+\left(1-\frac{2 m}{r}\right)\left(d x^{5}\right)^{2}+\frac{d r^{2}}{1-2 m / r}+r^{2} d \Omega_{2}^{2}
$$

This is simply the product of the Euclidean Schwarzschild solution with a trivial time direction, and is a completely nonsingular geometry. It describes a neutral "bubble": points $r<2 m$ are excised from spacetime, but this is done in a nonsingular way-if the periodicity of $x^{5}$ is chosen to be $8 \pi m$, the internal space smoothly closes at $r=2 m$. In the reduced description, it corresponds to a pointlike naked singularity, with Arnowitt-Deser-Misner (ADM) mass equal to $m / 2$.

Notice that the Schwarzschild solution, and all the electrically charged black holes can in turn be obtained by boosting the neutral bubble with velocity $v>1$. In a sense, the Schwarzschild solution and the neutral bubble are at opposite endpoints of the electrically charged spectrum. This will also be true when we add magnetic charge.

The general static, spherically symmetric solution with both electric and magnetic charges can be found in different parametrizations in [14-16], and we will use them to study the most general static spheric bubbles. We find it convenient to use the parametrization in [16]. This is given in terms of the two boost parameters applied to the Schwarzschild solution with mass $m$, and it is more closely related to the analysis above. For completeness, we discuss in Appendix A how the bubbles appear when the metric coefficients are expressed directly in terms of the physical parameters $M, P, Q$, and the scalar charge as in [15].

The five-dimensional geometry of the solutions is [16]

$$
\begin{gathered}
d s_{(5)}^{2}=\frac{X}{Y}\left(d x^{5}+2 A_{\mu} d x^{\mu}\right)^{2}-\frac{f}{X} d t^{2}+\frac{Y}{f} d r^{2}+Y d \Omega_{2}^{2}, \\
A_{t}=Q \frac{r+2 m}{X}, \quad A_{\varphi}=P \cos \theta,
\end{gathered}
$$

where

$$
\begin{gathered}
X=(r+2 m)\left(r+2 m \cosh ^{2} \delta_{Q}\right), \\
Y=r^{2}+2 m\left[\left(2-\cosh ^{2} \delta_{Q}\right) \cosh ^{2} \delta_{P}+\cosh ^{2} \delta_{Q}\right] r \\
+4 m^{2} \cosh ^{2} \delta_{P}, \\
f=r(r+2 m),
\end{gathered}
$$

and the electric and magnetic charges and ADM mass are ${ }^{1}$

$$
\begin{gathered}
Q=m \sinh \delta_{Q} \cosh \delta_{Q} \cosh \delta_{P}, \\
P=m\left(2-\cosh ^{2} \delta_{Q}\right) \sinh \delta_{P} \cosh \delta_{P}, \\
M=\frac{m}{2}\left(\cosh ^{2} \delta_{Q}+\cosh ^{2} \delta_{P}-\sinh ^{2} \delta_{Q} \sinh ^{2} \delta_{P}\right) .
\end{gathered}
$$

Here $m$ corresponds to the mass parameter of the Schwarzschild solution used to generate the whole family. Extremal solutions correspond to limits where $m \rightarrow 0$, while sending a boost angle to infinity to keep the mass and some of the charges fixed. ${ }^{2}$ To avoid Taub-NUT Newman-UntiTamburino terms in the reduced metric we must keep $m \sinh 2 \delta_{Q} \tanh \delta_{P}=0$.

The horizons correspond to the zeros of $f$ that are not simultaneously zeroes of $X, Y$. For $\cosh ^{2} \delta_{Q} \geqslant 1$ there is always a horizon at $r=0$. These solutions are black holes, and therefore we will concentrate on solutions with electric boost velocity greater than 1 , i.e., $\cosh ^{2} \delta_{Q} \leqslant 0$. The form of the metric coefficients $g_{55}$ and $g_{r r}$ tells us that bubbles will appear when $f$ and $X$ have a common zero, which must also be bigger than the roots of $Y$ since these, in general, are singularities. However, just as in the magnetically neutral case, and for the same reasons, the metric will be singular if there is a nonvanishing electric term. Thus we shall set $\cosh \delta_{Q}=0$. Notice that the neutral solution obtained by setting the electric and magnetic charge to zero by making $\cosh \delta_{Q}=\cosh \delta_{P}=0$ is singular. Therefore we consider $\cosh ^{2} \delta_{P} \neq 0$.

Remarkably, when $\cosh \delta_{Q}=0$ we have $f=X$ and the fivemetric is the direct product of a "trivial" time with a Euclidean four-geometry. The latter is, in fact, the Euclidean Taub-NUT metric. It can be written in a more familiar way by shifting $r+2 m \cosh ^{2} \delta_{P} \rightarrow r$ and then identifying the magnetic charge $P$ as the Euclidean nut parameter:

$$
\begin{gathered}
d s_{(5)}^{2}=-d t^{2}+U(r)\left(d x^{5}+2 P \cos \theta d \varphi\right)^{2} \\
+\frac{d r^{2}}{U(r)}+\left(r^{2}-P^{2}\right) d \Omega_{2}^{2}, \\
U(r)=\frac{r^{2}-4 M r+P^{2}}{r^{2}-P^{2}}
\end{gathered}
$$

(we stress that the parameter $M$ here is the mass of the monopole, and not the usual "mass parameter' of the fourdimensional Taub-NUT metric, which is in fact $2 M$ ). We still have to impose further regularity conditions on this solution, but for the moment note that this covers all the static,

\footnotetext{
${ }^{1}$ Our conventions differ slightly from [16]: with our definitions, the Maxwell field is one-half of theirs, and our (ADM) mass is one-fourth of their parameter $M$. Also, what we call $\delta_{P}$ is their $\delta_{1}$, and our $m$ is their $\beta$.

${ }^{2}$ There is a family of neutral solutions [6], of which the Schwarzschild black hole and the neutral bubble are particular cases, that is not contained in general in [16]. However, neither these nor their boosted counterparts yield new regular solutions.
} 
asymptotically flat, spherically symmetric, regular solutions. They represent localized lumps of energy that are sources of a monopolar magnetic field.

The factorized form of the geometry (2.10) greatly simplifies the analysis of the regularity of the solutions. There are only two completely nonsingular instantons constructed out of the Euclidean Taub-NUT solution: the self-dual TaubNUT instanton, and the Taub-bolt instanton. The EguchiHanson metric can be obtained as a limiting case of TaubNUT, but, in the KK context, it does not yield an asymptotically flat reduced geometry.

The self-dual Taub-NUT solution corresponds to a minimal value for the mass, $M_{N}=P / 2$, and yields just the GPS monopole, which is located at the nut fixed point of $\partial_{5}$. If we allow the mass of the monopole to be greater than the minimal value $M_{N}$, then, as shown by Page [17], there will be a two-dimensional fixed point set of $\partial_{5}$ (a spherical bolt) at the biggest root of $U(r), r_{+}=2 M+\sqrt{4 M^{2}-P^{2}}$. A conical singularity at the bolt can be avoided by choosing $M=5 P / 8$. The metric (2.10) with

$$
U_{B}(r)=\frac{\left(r-r_{+}\right)\left(r-r_{-}\right)}{r^{2}-P^{2}}, \quad r_{+}=2 P, \quad r_{-}=\frac{P}{2},
$$

is referred to as the "Taub-bolt" solution. It is not self-dual, and is topologically $\mathrm{CP}^{2}-\{0\}$. The point that has been removed from $\mathrm{CP}^{2}$ is "infinity" $(r \rightarrow \infty)$.

For all other values of $M>P / 2, M \neq 5 P / 8$, we obtain a conical singularity in the internal direction. This is a very mild singularity, and we could change the periodicity of $x^{5}$ to be $\Delta x^{5}=4 \pi r_{+}$so as to avoid the conical singularity at the bolt, but at the same time we should accordingly change the periodicity of $\varphi$ to $\Delta \varphi=\pi r_{+} / P$ to avoid the Dirac string singularities. In turn, this would result in a conical defect

$$
\delta=2 \pi\left(1-\frac{r_{+}}{2 P}\right)
$$

threading the monopole along the axes $\theta=0, \pi$. For $P / 2<M<5 P / 8$ the defect is a string, whereas for $M>5 P / 8$ it is a strut. Therefore, the former case can represent, if not new particlelike solutions, configurations of bolt monopoles threaded with cosmic string vortices, reminiscent of similar black hole/cosmic string configurations considered in [18].

It is interesting that the GPS monopole appears as an extremal limiting case of two kinds of objects: the magnetic black holes and the magnetic bubbles. The black holes can be regarded as thermally excited states of the monopole, to which they decay by emitting Hawking radiation. The bolt monopole is likely to correspond to another type of excited state, but since it has no horizon it does not emit Hawking radiation. However, it is known to be classically unstable, since the spectrum of fluctuations around the Euclidean Taub-bolt metric contains a negative mode [19], as does the Euclidean Schwarzschild solution. It has been speculated [8] that bolt monopoles should evolve to form a black hole, which would then decay to the GPS monopole. In any case, the most important restriction on the possible relevance of bolt monopoles comes from the fact that fermions rule them out, since the topology $\mathrm{CP}^{2}-\{0\}$ does not admit any spin structure.

An interesting outcome of the analysis in this section is the place we have found for the bubbles among the general spectrum of dyonic solutions. This will allow us to extend to bubbles the study of some processes involving black holes. Before that, we will analyze some striking features of the bubble solutions when the signature of the internal dimension is reversed.

\section{TWO-TIMING MONOPOLES}

As we saw in the introduction, given a solution to ordinary KK theory we can easily obtain another solution corresponding to a two-timing theory. However, in general, their properties, such as the nature of their singularities, are markedly different.

It is straightforward to see that, upon the continuations (1.2), (1.3), solutions with electric charge have, again, curvature singularities. On the other hand, the magnetic bubbles we have just described turn into products of the Lorentzian Taub-NUT solution with a trivial time:

$$
\begin{gathered}
d s_{(5)}^{2}=-d t^{2}-U(r)\left(d x^{5}+2 P \cos \theta d \varphi\right)^{2} \\
+\frac{d r^{2}}{U(r)}+\left(r^{2}+P^{2}\right) d \Omega_{2}^{2}, \\
U(r)=\frac{r^{2}-4 M r-P^{2}}{r^{2}+P^{2}},
\end{gathered}
$$

which is a solution with full five-dimensional signature $(--+++)$. After reduction, this describes an object with (imaginary) magnetic charge $P$ and mass $M$.

The metric (3.1) has singularities at

$$
r=r_{ \pm}=2 M \pm \sqrt{P^{2}+4 M^{2}} .
$$

Notice that $r_{+}>0$ and $r_{-}<0$. There is now an important difference with the one-timing solutions. As before, the length of the internal space generated by $x^{5}$ goes to zero at $r_{ \pm}$, but, as pointed out in [20], spacetime is easily extended through these surfaces, in a way analogous to the extension through a Lorentzian horizon. We summarize the causal structure of the maximal extension: (1) In the region $r_{-} \leqslant r \leqslant r_{+}$, the coordinate $r$ is timelike and there are no closed timelike curves (CTC's); (2) in the regions $r \leqslant r_{-}$and $r \geqslant r_{+}$, the coordinate $x^{5}$ is timelike and there are (microscopic) CTC's.

Thus, the surfaces $r=r_{ \pm}$are in fact chronology horizons. At $r=r_{ \pm}, U(r)=0$ and thus the "length" of the "internal space" generated by $x^{5}$ goes to zero there. Notice that the microscopic bubble region (1) connects two asymptotically flat regions.

Thus, we see that our two-timing Kaluza-Klein monopole can also be regarded as a "bubble" of Kleinian signature, which exists for all external time, and which is bounded from our part of the universe (where things are effectively Lorentzian) by a horizon at $r=r_{+}$. Observers "far away" from the source $\left(|r| \gg r_{ \pm}\right)$will see a magnetic monopole. As we approach $r_{+}$(from $r>r_{+}$), the size of the internal space 
shrinks to zero at $r=r_{+}$. As we move into the region $r_{-}<r<r_{+}$we see that the internal space is now spacelike. The "time" that used to live in the internal space is "uncompactified" in the region $r_{-}<r<r_{+}$, and so this region (to a five-dimensional observer, the region is too small to be detected by a four-dimensional observer) would look like a "bubble" of Kleinian signature, i.e., in the ill-defined effective "four-dimensional theory," the metric seems to change signature from $(--++)$ to $(-+++)$ as we move from the region $r_{-}<r<r_{+}$to the region $r>r_{+}$.

The magnetic charge of these solutions is, as we saw in the introduction, imaginary, and like-charged monopoles attract, rather than repel each other. No nonsingular neutral two-timing bubble exists: the magnetic charge $P$ is essential in allowing the extension of the metric through the interior of the bubble by preventing the formation of a curvature singularity inside it. Thus, the neutral solution with $P=0$ contains the usual Schwarzschild singularity at $r=0$. There are more respects in which the spectrum of two-timing bubbles differs from ordinary one-timing bubbles. For the latter, there is a state with minimal nonvanishing mass. This is the GPS monopole, which is extremal and saturates a Bogomolnyi bound. No extremal state exists for two-timing monopoles, and two-timing multimonopole static configurations necessarily contain conical singularities reflecting the presence of net forces between them.

There are more differences between both classes of objects. The background geometry of two-timing monopoles admits fermion structures. More strikingly, in the two-timing case the value of $M$ is completely unrestricted, and we can perfectly well set $M=0$. Thus we find a regular massless monopole. This case is also especially interesting since the two different regions $r>P$ and $r<-P$ connected by the Kleinian bubble are isometric under $r \rightarrow-r$.

In the massive case the isometry is broken. We can also have negative mass objects. This is surprising, since negative mass usually implies naked singularities. These are certainly present in the four-dimensional description of these monopoles, but the full solutions are regular. The significance of these negative mass solitons is unclear, but the apparent absence of a lower energy bound is probably one more indication of the pathological behavior of these theories.

The classical stability of these two-timing solutions cannot be inferred from that of their one-timing counterparts, and this remains an interesting problem. The fact that likecharges attract each other suggests that these monopoles would tend to a minimal size. Let us use this admittedly nonrigorous criterion to analyze which monopole configuration is likely to be more stable. The monopoles have two "mouths," one at $r=r_{+}$, the other at $r=r_{-}$. The total area of both mouths is $A \propto\left(r_{+}^{2}+r_{-}^{2}+2 P^{2}\right)=16 M^{2}+4 P^{2}$, so that, for fixed $P$, the minimal value is attained when $M=0$. This would suggest that the massless monopole might be, at least, more stable than the massive states, with masses of either sign. Whether these solutions can evolve to a black hole state is not clear, given the strange properties of black holes in these theories [13].

It is of some interest to calculate geodesic motion in the background of the two-timing monopole. To this end, note that from the metric (3.1) we recover, via the action principle, the following Lagrangian:

$$
\begin{aligned}
L= & -\dot{t}^{2}-U(r)\left(\dot{x}_{5}+2 P \cos \theta \dot{\varphi}\right)^{2}+\frac{1}{U(r)} \dot{r}^{2} \\
& +\left(r^{2}+P^{2}\right)\left(\dot{\theta}^{2}+\sin ^{2} \theta \dot{\varphi}^{2}\right)
\end{aligned}
$$

where the overdot denotes differentiation relative to any affine parameter $s$. As usual, for photons $L=0$ whereas for massive particles $L>0$. Now, $L$ is cyclic in $t, x_{5}$, and $\varphi$, and so we obtain three constants of motion:

$$
\begin{gathered}
\varepsilon=\dot{t}=\text { "energy" } \\
\omega=\left(r^{2}+P^{2}\right) \dot{\varphi}=\text { " angular momentum" } \\
\delta=U(r) \dot{x}_{5}=\text { " } \text { five-dimensional velocity". }
\end{gathered}
$$

The five-dimensional velocity is interpreted by the fourdimensional observer as electric charge.

For simplicity, we restrict our attention to the motion of test particles in the $\theta=(\pi / 2)$ plane, i.e., $\dot{\theta}=0$ for all times. Thus,

$$
L=-\dot{t}^{2}-U(r) \dot{x}_{5}^{2}+\frac{1}{U(r)} \dot{r}^{2}+\left(r^{2}+P^{2}\right) \dot{\varphi}^{2} .
$$

To be concise, we will only consider the motion of light-like particles, "photons"; the timelike case is very similar. Thus, assuming $L=0$ we see that the Lagrangian assumes the form

$$
\left(\frac{d r}{d \varphi}\right)^{2}=U(r)\left(r^{2}+P^{2}\right)\left[\frac{\varepsilon^{2}}{\omega^{2}}+\frac{\delta^{2}}{U(r) \omega^{2}}-1\right] .
$$

Radial motion of the photon is possible only if $(d r / d \varphi)^{2}>0$, i.e., if and only if

$$
\varepsilon^{2}>\omega^{2}-\frac{\delta^{2}}{U(r)} .
$$

A natural question is whether or not it is possible for a photon to escape out to infinity $(r=+\infty)$ from the monopole "core" (i.e., $r=r_{+}$). Of course, the answer to this question is trivial once one realizes that at $r=r_{+}, U\left(r_{+}\right)=0$. That is, the above inequality becomes

$$
\varepsilon^{2}>\omega^{2}-\infty,
$$

which is trivially satisfied for any values of the energy and angular momentum, as long as the "charge" $\delta$ is nonvanishing (even when $\delta$ is vanishing we can just set the angular momentum to zero). Thus, any photon starting in the core will always make it out to $r=\infty$. Likewise, since any photon beginning in the asymptotic region will trivially satisfy $\varepsilon>-\infty$, any such particle can fly through the monopole core and emerge in the other asymptotic region.

From the point of view of quantum scattering, this presumably means that the monopole is stable to quantum corrections as long as the flux from one asymptotic region is exactly compensated by the flux from the other region. However, as we discussed above, the two asymptotic regions are isometric if and only if $M=0$. This would seem to provide 
further support to the conjecture that the $M=0$ state would be "stable," and that massive states would want to "settle down', to $M=0$.

However, the massless two-timing monopole is neither extremal nor does it saturate any Bogomolnyi-type bound. Therefore we do not expect to find that the background admits $N=1$ supersymmetry. In other words, there should not exist any covariantly constant spinor field $\epsilon$ (by covariantly constant we mean constant relative to the Levi-Civita connection on Taub-NUT). To see that this is true simply recall that the only Lorentzian manifolds admitting covariantly constant spinor fields are the $p p$ waves; however, all $p p$ waves have type $N$ curvature, whereas Taub-NUT is of type $D$. In view of this, the precise meaning of our vague arguments about the stability of the solutions is at present unclear.

\section{PAIR CREATION OF NEUTRAL BUBBLES}

\section{A. $C$ metric for accelerating bubbles}

A number of ways of pair producing black holes have been studied in recent years. In Sec. II we have seen that Kaluza-Klein black holes and bubbles form part of the same family of dyonic solutions. This will allow us to obtain an instanton describing pair creation of neutral bubbles. The bubbles will appear at the ends of a cosmic string that snaps into two pieces; after that, they accelerate away pulled by the string tension. Notice that the same argument that allows a topologically stable string vortex to end in a black hole $[18,21]$ can be extended to a neutral bubble: the nontrivial topology $\mathbf{R} \times S^{2} \times \mathbf{R}^{2}$ of the neutral bubble allows to construct a nonsingular Wu-Yang fibration of the gauge field carried by the cosmic string, so that it is well defined everywhere.

How do we construct the metric that describes two accelerating bubbles? The key is the relation that we have found between electrically charged KK black holes and neutral bubbles. For our present purposes this relation takes a more convenient form if we first rewrite the metric (2.1) by making $r+2 m \sinh ^{2} \delta_{Q} \rightarrow r$, and renaming

$$
r_{+} \equiv 2 m \cosh ^{2} \delta_{Q}, \quad r_{-} \equiv 2 m \sinh ^{2} \delta_{Q} .
$$

The metric for static electric black holes is then

$$
\begin{aligned}
d s^{2}= & \left(1-\frac{r_{-}}{r}\right)^{-1}\left(d x^{5}+\frac{2 Q}{r} d t\right)^{2}-\left(1-\frac{r_{+}}{r}\right) d t^{2} \\
& +\frac{1-r_{-} / r}{1-r_{+} / r} d r^{2}+r^{2}\left(1-\frac{r_{-}}{r}\right)^{2} d \Omega_{2}^{2},
\end{aligned}
$$

with

$$
Q=\frac{\sqrt{r_{+} r_{-}}}{2} .
$$

The radii $r_{ \pm}$must satisfy $r_{+}-r_{-} \geqslant 0$, and $r_{+} r_{-} \geqslant 0$. The mass of the black holes is

$$
M=\frac{1}{2}\left(r_{+}-\frac{r_{-}}{2}\right) .
$$

The (singular) electric bubbles correspond to $0 \leqslant-r_{+}<-r_{-}$, and the neutral bubble appears in the limiting case where $r_{+}=0$. In this case, the mass of the bubble is $M=-r_{-} / 4>0$, and it is located at $r=0$.

In [22] a dilaton $C$ metric describing magnetically charged black holes accelerating apart is given. We particularize to the $\mathrm{KK}$ case, and perform a generalized duality transformation

$$
\begin{gathered}
\widetilde{F}_{\mu \nu}=\frac{1}{2} e^{-2 \sqrt{3} \phi} \epsilon_{\mu \nu \rho \sigma} F^{\rho \sigma}, \\
\phi \rightarrow-\phi,
\end{gathered}
$$

while leaving the effective four-metric unchanged. In this way we obtain the following five-dimensional metric describing electrically charged accelerating black holes:

$$
\begin{aligned}
d s^{2}= & \frac{F(x)}{F(y)}\left(d x^{5}+2 Q y d t\right)^{2}+\frac{1}{A^{2}(x-y)^{2}}\left[F ( x ) \left(G(y) d t^{2}\right.\right. \\
& \left.\left.-\frac{F(y)}{G(y)} d y^{2}\right)+F(y)^{2}\left(\frac{d x^{2}}{G(x)}+\frac{G(x)}{F(x)} d \varphi^{2}\right)\right]
\end{aligned}
$$

where $Q=\sqrt{r_{+} r_{-}} / 2$, and

$$
\begin{gathered}
F(\xi)=1+r_{-} A \xi \\
G(\xi)=1-\xi^{2}-r_{+} A \xi^{3} .
\end{gathered}
$$

We will see below how this metric can also describe accelerating electric (singular) or neutral bubbles, but it is first convenient to briefly analyze the physical interpretation of the metric when the parameters are chosen so as to describe black holes.

The root of $F$ is $\xi_{1}=-1 /\left(r_{-} A\right)$, and $G$ has three roots $\xi_{2}, \xi_{3}, \xi_{4}$. It is convenient to analyze them for small $r_{ \pm} A$. Then $\xi_{1}$ is large and negative, as is also $\xi_{2} \approx-1 /\left(r_{+} A\right)$. The other two roots are of order 1: $\xi_{3} \approx-1, \xi_{4} \approx+1$. When $r_{+} \geqslant r_{-} \geqslant 0$ their physical meaning can be obtained by considering the following limits.

(1) We blow up the region near $y=\xi_{1}, \xi_{2}$ by making $y=-1 /(r A)$, and $A \rightarrow 0$. In this case, with the additional change $x=\cos \theta$ and rescaling $t \rightarrow A t$, the metric reduces to (4.2). Alternatively, instead of saying that we go close to the black holes, one could interpret this as the static limit of the metric, where the acceleration parameter is set to zero and the conformal factor $A^{-2}(x-y)^{-2}$ in front of the four metric is removed.

(2) While still keeping $r_{ \pm} A \ll 1$, consider now the region near $y=\xi_{4} \approx 1$. This root corresponds to an acceleration horizon, since in this case, with $F(y), F(x) \approx 1$, the metric becomes that of Rindler space (though written in unusual coordinates), crossed with a trivial internal space. Points of constant $y, x$ are moving with uniform acceleration. It can also be interpreted as a small particle limit.

This interpretation holds when $y$ lies in the range $-1 /\left(r_{-} A\right)<y<x$. The endpoints of this interval are singular, and correspond to the singularity inside the black hole and asymptotic infinity.

It is also clear that we must keep the coordinate $x$ between the two order 1 roots of $G(x), \xi_{3} \leqslant x \leqslant \xi_{4}$. Then $x$ plays the 
role of $\cos \theta$ in the angular $(x, \varphi)$ part of the metric, which is topologically a sphere $S^{2}$. However, this sphere is distorted, and can easily be seen to contain necessarily a conical singularity at one of the poles, reflecting the need for an accelerating force.

From our discussion of the static solution above, it is clear what to do to obtain accelerating bubbles. Singular electric bubbles appear for $0 \leqslant-r_{+}<-r_{-}$, and the regular neutral bubble corresponds to $r_{+}=0,-r_{-}=4 M$. In this case

$$
F(\xi)=1-4 M A \xi, \quad G(\xi)=1-\xi^{2},
$$

and all the roots are easily given: $\xi_{1}=-1, \xi_{2}=1$, $\xi_{3}=1 /(4 M A)$. Notice that the ordering of the roots has changed. Now we take $-1 \leqslant x \leqslant 1$ and $-\infty<y<x$. The axis $x=-1$ points to infinity, whereas $x=1$ points to the other black hole. The value $y=-1$ corresponds again to the acceleration horizon. But now, instead of a black hole horizon we find a bubble located at the zero of $F(y)^{-1}$, i.e., at $y \rightarrow-\infty$, where the size of the internal circles vanishes. This is just as expected, since by taking $y=-1 /(r A)$ and $A \rightarrow 0$ we recover the neutral bubble metric, with the bubble at $r=0$.

We now analyze in more detail the conical singularities that pull the bubble. The relevant part of the metric is

$$
{ }^{(2)} d s^{2}=\frac{d x^{2}}{1-x^{2}}+\frac{1-x^{2}}{F(x)} d \varphi^{2} .
$$

For $-1 \leqslant x \leqslant 1$ this is topologically $S^{2}$. If the periodicity of $\varphi$ is $\Delta \varphi$, there is a conical deficit at each of the poles $x= \pm 1$ given by

$$
\delta_{( \pm 1)}=2 \pi\left(1-\frac{\Delta \varphi}{2 \pi \sqrt{F( \pm 1)}}\right) .
$$

Clearly, it cannot be made zero simultaneously at both poles. We choose to keep regularity along the axis $x=1$ joining the black holes, so we take

$$
\Delta \varphi=2 \pi \sqrt{F(1)}
$$

A conical defect runs along the axis $x=-1$, i.e., from each black hole to infinity. We can interpret it as coming from a cosmic string with tension $\mu=\delta /(8 \pi)$, which is pulling the black holes apart. The mass and acceleration are related to the string tension by

$$
\mu=\frac{1}{4}\left(1-\sqrt{\frac{1-4 M A}{1+4 M A}}\right) \approx M A,
$$

where the last approximate equality is obtained for small $M A$ and reproduces Newton's law as expected.

We require the geometry to be regular at the bubble. To do so, we focus on the $\left(x^{5}, y\right)$ part of the metric, in the limit $y \rightarrow-\infty$. In this case, absence of conical singularities requires the period of $x^{5}$ to be

$$
\Delta x^{5}=16 \pi M .
$$

Finally, we study the possibility of constructing a Euclidean instanton describing pair production of these bubbles.
We Wick rotate $t \rightarrow i \tau$, and analyze what condition must be imposed on the Euclidean time period $\Delta \tau$ so as to avoid a conical singularity at the acceleration horizon $y=-1$. Note that there is no other horizon and the bubble has no prefixed temperature. Then it is always possible to attain regularity by setting

$$
\Delta \tau=\frac{4 \pi \sqrt{F(-1)}}{\left|G^{\prime}(-1)\right|}=2 \pi \sqrt{1+4 M A}
$$

This Euclidean solution describes the worldsheet of a cosmic string containing a loophole delimited by a circular bubble trajectory. The decay process is described as usual: take the initial configuration to be that of a cosmic string (i.e., a conical defect) with the required tension in flat space. The Euclidean $C$ metric above tends asymptotically to this configuration. Moreover, surfaces $\tau=$ const of the $C$-metric instanton have the same geometry as the $t=0$ section of the Lorentzian solution. Since their extrinsic curvature is vanishing, we can smoothly "glue" the Euclidean and Lorentzian sections and thus describe a process in which a cosmic string snaps and bubbles appear at its ends. The semicircular Euclidean bubble trajectory corresponds to the bubbles tunneling towards a configuration where they both have zero velocity; at that point, the transition to Lorentzian signature (real time) takes place, and the circular motion turns into uniformly accelerated hyperbolic trajectories for the pair of bubbles just created. This breaking of a cosmic string with formation of neutral bubbles at its ends provides an alternative to the processes where the string breaks by forming black holes [24].

It is interesting to notice that we have been able to construct a $C$-metric instanton for a neutral object. This is new: usually, charge is needed to lower the black hole horizon temperature to match the acceleration temperature. In this case, however, we have a neutral object with zero (more properly, nonfixed) temperature, and no such problem is present. Moreover, as far as we know, this is the first nonextremal pair creation instanton constructed for a theory with dilaton coupling parameter $a>1$. In these theories, the black hole temperature grows without limit as one approaches extremality, and this precludes the possibility of regularly matching the Euclidean time periodicity. Clearly, this obstruction disappears for bubbles.

One could also expect to be able to create neutral bubbles in a cosmological context, in a way similar to the nucleation of Schwarzschild black holes in a de Sitter universe [25]. However, it is very unlikely that a similar solution for bubbles exists within the framework of pure KK theory. Notice that a cosmological constant $\Lambda$ in five dimensions would yield, upon reduction, a four-dimensional Liouville-type potential $\Lambda e^{2 \phi / \sqrt{3}}$, which is inversely proportional to the length of the internal circles. This potential would diverge at the position of a bubble, where the internal space closes up. Such a divergence, however, would come entirely from the conformal factor in the metric and, even if somewhat undesirable, it might give no problems in the higher dimensional metric. We would expect to find a neutral bubble if we had a KK cosmological (de Sitter) solution containing an electrically charged black hole. The bubble would correspond to taking the electric charge to be zero in the way described in 
Sec. II. However, it has been argued in [26] that no such charged solutions exist for nontrivial Liouville-type dilaton potentials. In principle, this does not rule out completely the existence of neutral bubble cosmological solutions, since they could exist and not be related to any electric black hole spectrum, but this certainly seems unlikely.

\section{B. Euclidean actions and pair creation rate}

As usual, by continuing the solutions to Euclidean time, their action can be used to obtain thermodynamical properties or pair creation rates. Upon dimensional reduction, the five-dimensional action

$$
I=\frac{1}{16 \pi G_{5}} \int_{\tilde{\mathcal{M}}} \sqrt{|\widetilde{g}|} \widetilde{R}-\frac{1}{8 \pi G_{5}} \int_{\partial \mathcal{\mathcal { M }}} \sqrt{|\widetilde{h}|} \widetilde{K}
$$

(quantities with tildes will correspond to the 5D description) turns to

$$
\begin{aligned}
I= & \frac{1}{16 \pi G_{4}} \int_{\mathcal{M}} \sqrt{|g|}\left[R-2(\nabla \phi)^{2}-e^{-2 \sqrt{3} \phi} F^{2}\right] \\
& -\frac{1}{8 \pi G_{4}} \int_{\partial \mathcal{M}} \sqrt{|h|} K .
\end{aligned}
$$

The boundary $\partial \mathcal{M}$ is taken to be the one induced by obtaining the quotient of the five-dimensional boundary $\partial \widetilde{\mathcal{M}}$. Also, the five-dimensional and four-dimensional coupling constants are related by $G_{4}=G_{5} / \Delta x^{5}$. For exact solutions of the Kaluza-Klein equations, the volume term in Eq. (4.16) vanishes and only the boundary term remains. Dimensional reduction of this term yields

$$
\begin{aligned}
\sqrt{\mid \widetilde{h}} \widetilde{K} & =\sqrt{|h|} \frac{e^{-\phi / \sqrt{3}}}{\sqrt{|g|}} \partial_{\mu}\left(e^{\phi / \sqrt{3}} \sqrt{|g|} n^{\mu}\right) \\
& =\sqrt{|h|} K+\sqrt{|h|} n^{\mu} \partial_{\mu} \ln \left(e^{\phi / \sqrt{3}} \sqrt{g}\right),
\end{aligned}
$$

where $n$ is the normal vector to the boundary $\partial \mathcal{M}$ induced by the normal $\tilde{n}$ to $\partial \widetilde{\mathcal{M}}$,

$$
\tilde{n} \rightarrow e^{-\phi / \sqrt{3}} n .
$$

Thus we see that the result not only contains the extrinsic curvature boundary term in Eq. (4.17), but also a surface term involving the scalar field. The latter can be obtained from Eq. (4.17) on shell by substituting the scalar field equation of motion, as is done in [23].

We have remarked that the bubbles are, in the fourdimensional effective description, naked singular points. However, when computing the action one should not include an additional boundary surrounding the singularity, since this must not be introduced in five dimensions, where the geometry is regular. As we have just explained, once the boundary terms at infinity have been properly accounted for, the results should be the same if we work in either 5D or 4D.

From the Euclidean continuation of the static metrics in Sec. II we obtain their thermodynamical properties, such as the entropy. The static KK Schwarzschild solution and the neutral bubble correspond, in the Euclidean regime, to the same metric. However, the Schwarzschild black hole has the usual Bekenstein-Hawking entropy, whereas no entropy is associated to the bubbles. Of course, there is nothing paradoxical here, since the entropy depends on the choice of the periodic variable (Euclidean time) that is associated to the asymptotic temperature. For both the Euclidean continuation $t \rightarrow i \tau$ of the bubble solution (2.4) and Euclidean Schwarzschild $\times\left(d x^{5}\right)^{2}$, the action is ${ }^{3}$

$$
I=\frac{1}{2} m \Delta \tau
$$

However, for the bubble, $M_{B}=m / 2$, whereas for Schwarzschild $M_{S}=m$. Then, setting $\Delta \tau=\beta$,

$$
I_{\mathrm{bub}}=\beta M_{B}, \quad I_{\mathrm{Sch}}=\frac{1}{2} \beta M_{S} .
$$

The vector $\partial_{\tau}$ has a fixed point at the horizon for the Schwarzschild solution, but it acts freely on the bubble. In the latter case, $\beta$ and $M$ can be varied independently while remaining on shell. The usual reasoning then leads to zero entropy for the bubble, and Bekenstein-Hawking entropy $S=A / 4$ for the black hole. As we have stressed, for both solutions the only boundary is the surface at infinity.

The action of the analytically continued $C$ metric gives the dominant contribution $e^{-I}$ to the semiclassical bubble pair production by snapping strings. The calculation requires carefully matching the boundaries near infinity $x=y=\xi_{1}=-1$ of the $C$ metric and the reference background (flat space with a cosmic string), see e.g., [27]. Then, straightforward application of the formulas above for the action gives the result

$$
I=\frac{\pi}{4 A^{2}}\left|F^{\prime}(-1)\right| \sqrt{F(1) F(-1)}=\frac{\pi M}{A} \sqrt{1-16 M^{2} A^{2}} .
$$

This answer can also be easily obtained by decomposing the action as

$$
I=\beta H-\frac{1}{4} \Delta A,
$$

where $H$ is the physical Hamiltonian and $\Delta A$ is the difference in the areas of the acceleration horizons of the $C$ metric and the reference background [27]. As it turns, $H=0$, and the difference in acceleration horizon areas is found to account for the entire action (4.22). Again, no boundary conditions have to be imposed at the four-dimensional singularity.

The exact relation between $A$ and $\mu$ can be inverted to express the action in terms of only $M$ and $\mu$ :

$$
I=\frac{\pi M^{2}}{\mu} \frac{1-4 \mu}{1-2 \mu} .
$$

For small $\mu$, the leading term is the same as for string breaking with monopole or black hole formation. Subleading cor-

\footnotetext{
${ }^{3}$ From now on we set $G_{4}=1$. Thus $\Delta x^{5}$ does not appear in Eq. (4.20).
} 
rections are due to graviton and scalar exchange between different points of the Euclidean circular trajectory of the bubble.

\section{PAIR CREATION OF MAGNETIC BUBBLES AND DYSON'S INSTABILITY}

Now that we know how to pair create neutral bubbles, it would be natural to try and look for pair creation of magnetic bubbles. Such a process could be readily described if we had a KK $C$ metric for dyonic black holes. Our analysis in Sec. II shows that magnetic bubbles are easily obtained from dyonic black hole solutions by sending the electric charge to zero in the appropriate way, i.e., by taking an infinite velocity boost in the internal direction. As we have just seen in the previous section, this method certainly works for $C$-type metrics. Thus, we believe that a $C$ metric for magnetic bubbles can be constructed.

However, the Kaluza-Klein dyonic $C$ metric is likely to be very complicated, since it must combine the intricacies of both the static dyonic solutions (to which it must reduce in the limit $A \rightarrow 0$ ), and of the $C$ metrics. The full dyonic $C$-metric solution must simplify greatly when particularized to describe accelerating magnetic bubbles, so one could try to find first the metric for the latter, which, by the way, is the situation of interest to us now. Even with this simplification, the solution is probably not as simple as any of the known $C$ metrics, and we have not found it yet. Some steps towards its construction are described in Appendix B.

Although we do not have the explicit form of the solution, we can semiquantitatively describe several features it must certainly have. Just like in the neutral case, this $C$ metric must contain conical singularities pulling apart the bubbles. Since the bubbles are magnetically charged, these singularities can be removed by introducing a background magnetic field $B$ asymptoting to the Kaluza-Klein Melvin solution, which then provides the required force. In Kaluza-Klein theory there is a simple way to do this: when identifying points in the internal direction, introduce a twist along the axial rotation axis [22]. The solution thus obtained could be used to describe a new decay mode of magnetic fields in Kaluza-Klein theory. To leading order, the action of the instanton should be given by the Schwinger value

$$
I=\frac{\pi M^{2}}{P B}+\cdots,
$$

and would be smallest for the minimal mass bubble, i.e., the extremal GPS monopole. Also, the entropy enhancement factors of nonextremal black holes should be absent.

Having a metric that describes two accelerating magnetic bubbles, then, as we have seen, we could easily obtain from it a metric corresponding to two accelerating two-timing monopoles. In particular, the massless case is of great interest, since no force is needed to accelerate the monopoles, and therefore, no conical singularities should appear. That this no-external-force condition can actually be met for massless objects has been explicitly verified for accelerating massless black holes in $[13,28]$. The anti-Maxwell theories considered in [13] include the two-timing KK theory for a specific value of the dilaton. However, the attempt at constructing a regular
Euclidean instanton describing Dyson's pair creation of antiMaxwell massless black holes failed. There are no Einsteinanti-Maxwell-dilaton extremal black holes, and it is not possible to lower down the black hole horizon temperature to a value small enough to match the acceleration temperature. ${ }^{4}$ Remarkably enough, this obstruction should be absent for the monopole bubbles, since they have no horizon and no prefixed temperature. Thus, the two-timing vacuum would not decay by production of massless black holes, but rather by forming zero mass monopole pairs.

For a general massive two-timing monopole, if no background field is introduced and, therefore, the monopoles are pulled by strings, ${ }^{5}$ we expect the action of the instanton to be given by the usual effective low energy result

$$
I=\frac{\pi M^{2}}{\mu}+O\left(P^{2}\right)=\frac{\pi M}{A}+O\left(P^{2}\right)
$$

where, we recall, $P$ is the magnetic charge. The massless monopole configuration is smoothly connected to the positive mass solutions. However, in the massless limit the leading term $\pi M / A$ in the action vanishes, and the qualitative behavior of creation rates changes. It is no longer of the usual exponentially damped Schwinger form, and is dominated by the $O\left(P^{2}\right)$ terms, which without explicit computation we cannot determine. However, these terms come from Coulomb corrections due to graviton, photon, and scalar exchange between different points of the circular trajectory. ${ }^{6}$ For, e.g., gravitating monopoles, this term is $\pi P^{2} / 2$, which is positive due to particle-antiparticle attraction. Hence, for two-timing monopoles it would be reasonable to expect this term to be $-\pi P^{2} / 2$, and we would find negative action in the massless case. In fact, recently a Euclidean $C$-metric instanton with massless black holes has been found to have precisely an action of this kind [28]. The repulsion between two oppositely charged two-timing monopoles makes it not very surprising to find an enhanced (instead of suppressed) pair creation rate. Pair creation of massless two-timing monopoles would thus imply a crass instability of the twotiming vacuum.

On the other hand, we could also consider a solution with accelerating negative mass monopoles. This solution can asymptote to the two-timing flat vacuum if we allow for a conical deficit in between the monopoles: the singularity would pull them together, but, since they have negative mass, they accelerate in the opposite direction. The conical deficit can operationally be replaced by a cosmic string such as in [30], and in this way we would find a decay of the two-timing vacuum by spontaneously producing cosmic strings with negative mass monopoles at their ends.

\footnotetext{
${ }^{4}$ It has been pointed out by Hawking that this absence of an extremal limit is not as odd as it may appear: in anti-Maxwell theory, the photons emitted by a black hole have negative energy, and therefore a black hole should increase its mass by radiating them out [29].

${ }^{5}$ The following heuristic discussion can be easily generalized to include pair creation in external fields.

${ }^{6}$ For black holes, the entropy enters also at this order.
} 
Notice that in two-timing decay processes the tunneling takes place through a path that, though effectively Euclidean in four dimensions, is Lorentzian in the full five-dimensional picture. In this case it is not completely clear how the classical action contributes to the leading terms of the decay rate. A possible resolution is to simply take the point of view of the effective four-dimensional description and write $\Gamma \sim e^{-I}$.

As usual, assuming a supersymmetric vacuum will rule out the decay of the vacuum via the new $C$-metric instanton that we have attempted to find here. More explicitly, the instanton will simply be connected and so the only spin structure allowed will be the one for which the fermions satisfy aperiodic boundary conditions as they are parallel propagated around the internal space. However, for supersymmetry we would require the fermions to be periodic on the internal space, and therefore it would not be possible to match the fermion structures when trying to construct the pair creation instanton. As in [4], this is the most natural mechanism for suppressing this pathological behavior.

\section{DISCUSSION AND CONCLUSIONS}

The bubble solutions of the form $\left(-d t^{2}\right)$ $\times$ Euclidean-Taub-NUT in Sec. II were already constructed some time ago $[5,6]$. However, our approach to constructing them by identifying the solutions in the parameter space of dyonic black hole solutions is much more general. It has allowed us to extend the class of pair creation processes studied till now. Interestingly, we have found that nonextremal pair creation instantons are indeed possible in a theory with dilaton coupling $a>1$. Another application of our method for finding bubbles is to other theories containing internal dimensions. In this way one can find bubbles-including also bubble strings - as solutions of the low energy effective equations of string theory, and of other higher dimensional theories. These will be discussed elsewhere. It should be kept in mind that, like many extended black objects, the (onetiming) bubbles studied here suffer from classical instabilities.

Given the finite size of bubbles, it is natural to ask whether rotating bubbles exist. However, this does not seem likely to occur, at least in 5D KK. If we consider the rotating dyonic solutions of this theory [32], then any way of eliminating the electric charge to obtain bubbles (such as taking boosts $v \rightarrow \infty$ ) also sends the angular momentum of the solution to zero. In fact, the bubble solutions thus obtained take the form of $\left(-d t^{2}\right) \times$ Euclidean-Kerr-Taub-NUT. These do not correspond to rotating bubbles, but, rather, to objects in background magnetic fields [31]. When the "Euclideanrotation" parameter $\Omega$ is small as compared to the inverse compactification scale, the four-dimensional description is that of a distorted bubble in a background magnetic field. The bubble has a magnetic dipole moment, in addition to the monopolar charge it may already have. On the other hand, if $\Omega$ is comparable to the inverse compact radius, then the appropriate picture is in terms of two GPS monopoles at antipodal points of the Euclidean $S^{2}$ bolt, with, in general, charges of different magnitude. In all these configurations, conical singularities appear so as to keep the whole set static.

A peculiar outcome of our analysis is the massless mono- pole in the two-timing theory. It is likely that massless objects are a rather general feature of theories with internal times. Usually, the mass and charges are expressable as sums and products, respectively, of several characteristic radii, such as in Eqs. (4.3) and (4.4). The imaginary charge associated with an internal time may be obtained by changing the sign of one of these radii, and this, in turn, may lead to regions of parameter space where the mass can be set to zero.

On the other hand, classical massless black hole solutions have been recently found in the context of string theory [33]. They exhibit a number of peculiar features, most of them associated to the fact that they are nakedly singular [28]. Our massless monopoles exhibit also a singularity in four dimensions, but this is harmless since we know how to handle it by going to the regular five-dimensional manifold. Another significant difference is that, whereas the stringy massless holes are supersymmetric, the two-timing massless monopoles are not. As a consequence, they are expected to acquire mass by quantum corrections. Given the presence of negative mass states, it is not clear what the quantum corrected mass spectrum of monopoles would be. As we have argued, the massless monopole could be stabilized due to the symmetry between the two asymptotic regions it connects. However, the quantization of the two-timing theory is certainly bound to exhibit all kinds of pathologies.

Finally, we have presented evidence that theories with internal time directions can give rise to pair production of massless particles out of the vacuum. This evidence is based on the following facts: (i) The 5D KK theory contains a nonsingular massless monopole, which can be obtained by appropriately choosing parameters in the general dyonic solution; (ii) dilaton $C$ metrics for both electric and magnetic black holes are known, so we expect that a similar metric for dyonic black holes must exist; (iii) we have explicitly shown that it is possible to construct, using the $C$ metrics already known, instantons for pair production of bubbles.

Thus, theories with extra times are expected to suffer from an instability that in some sense is even worse than those previously known. Pathologies caused by negative norm fluctuations of the internal dimension can be kept below a certain level by, for example, taking the internal radius to be sufficiently small. On the other hand, the instability of the ordinary KK vacuum described in [4] is suppressed for large internal radius. ${ }^{7}$ In contrast, production of massless two-timing monopoles is, as we argued, probably enhanced. Work towards the explicit construction of the corresponding $C$ metric, as well as the more general KK dyonic $C$ metric, is in progress.

\section{ACKNOWLEDGMENTS}

A.C. was supported by Grant No. NSF PHY94-07194. R.E. was partially supported by FPI through MEC (Spain), and by Grant Nos. CYCIT AEN-93-1435 and UPV 063.310EB225/95.

\footnotetext{
${ }^{7}$ Notice that this decay mode cannot be generalized to the twotiming theory, since by Wick rotating the internal direction of the instanton we find the 5D Lorentzian Schwarzschild solution, which is not regular.
} 


\section{APPENDIX A}

For some purposes, it is useful to write the solution (2.5), (2.7) as it is done in [15], in terms of the more physical parameters $M, P, Q$, and the scalar charge $\Sigma$. The latter is obtained as $\phi \rightarrow \Sigma / r+O\left(r^{-2}\right)$ and is not an independent parameter, but rather it must satisfy

$$
\frac{2}{3} \Sigma=\frac{Q^{2}}{\Sigma-\sqrt{3} M}+\frac{P^{2}}{\Sigma+\sqrt{3} M} .
$$

The functions (2.7), after a linear shift of $r$, are given by

$$
\begin{gathered}
X=(r-\Sigma / \sqrt{3})^{2}+\frac{2 Q^{2} \Sigma}{\Sigma-\sqrt{3} M}, \\
Y=(r+\Sigma / \sqrt{3})^{2}+\frac{2 P^{2} \Sigma}{\Sigma+\sqrt{3} M}, \\
f=(r-M)^{2}-\left(M^{2}+\Sigma^{2}-Q^{2}-P^{2}\right),
\end{gathered}
$$

and

$$
\begin{gathered}
A_{t}=Q \frac{r+\Sigma / \sqrt{3}}{X}, \\
A_{\varphi}=P \cos \theta .
\end{gathered}
$$

This representation has the advantage that the electricmagnetic duality (4.5) of the four-dimensional description is evident from the symmetry under $Q \leftrightarrow P, \Sigma \rightarrow-\Sigma$.

At first sight, it is not clear how to obtain the bubble sector of the solution, since if we directly set $Q=0$ we only find the magnetically charged black holes. However, a different solution is obtained if we send $Q \rightarrow 0$ and simultaneously $\Sigma \rightarrow \sqrt{3} M$, while keeping the ratio $Q^{2} /(\Sigma-\sqrt{3} M)$ finite and fixed by Eq. (A1). In this limit we find

$$
\begin{gathered}
X=f=\left(r-M-\sqrt{4 M^{2}-P^{2}}\right)\left(r-M+\sqrt{4 M^{2}-P^{2}}\right), \\
Y=(r+M-P)(r+M+P) .
\end{gathered}
$$

Shifting now $r+M \rightarrow r$ we find the same metric as in Eq. (2.10).

\section{APPENDIX B}

In this appendix we find a unified form for the several known limits that the $C$ metric for magnetic bubbles must satisfy. The full solution has not been found yet, but we think that the results presented here may be useful for further investigations.

The KK $C$ metric for magnetic bubbles will depend on three parameters, namely $r_{+}, r_{-}, A$. It must certainly satisfy the following limits: (1) For vanishing $r_{+}$or $r_{-}$it must reduce to the neutral bubble $C$ metric; (2) for $r_{+}=r_{-}$it must reproduce the $C$ metric describing creation of GPS monopoles [23]; (3) for $A \rightarrow 0$ it must reduce to the static general Taub-NUT solution across a trivial time.

Of course, these metrics need not be reproduced in precisely the form given above or in [23], and some coordinate changes may be needed. We will rewrite all these metrics in a way that a unified form of all of them is possible.

Start first with the static limit, i.e., Taub-NUT $\times\left(-d t^{2}\right)$. Writing it as

$$
\begin{aligned}
d s^{2}= & -d t^{2}+\frac{\left(r-r_{+}\right)\left(r-r_{-}\right)}{r^{2}-r_{+} r_{-}}\left[d x^{5}+2 \sqrt{r_{+} r_{-}} \cos \theta d \varphi\right]^{2} \\
& +\frac{r^{2}-r_{+} r_{-}}{\left(r-r_{+}\right)\left(r-r_{-}\right)} d r^{2}+\left(r^{2}-r_{+} r_{-}\right) d \Omega_{2}^{2}
\end{aligned}
$$

we change to the coordinate

$$
y=-\frac{1}{\left(r+r_{+}\right) A}
$$

(other choices are possible, such as $y=-1 /(r A)$ or $y=-1 /\left[\left(r \pm r_{-}\right) A\right]$, but this one is quite convenient since it will allow for the simplest form of the off-diagonal term in the $C$ metrics with magnetic charge). In this static metric the parameter $A$ has no special meaning, and simply has the dimensions of an inverse length.

Then, the metric (B1) is

$$
\begin{aligned}
d s^{2}= & \frac{H(y) E(y)}{J(y)}\left(d x^{5}+2 \sqrt{r_{+} r_{-}} \cos \theta d \varphi\right)^{2}-d t^{2} \\
& +\frac{J(y)}{H(y) E(y)} \frac{d y^{2}}{A^{2} y^{4}}+\frac{J(y)}{A^{2} y^{2}} d \Omega_{2}^{2},
\end{aligned}
$$

where we have defined the following polynomial functions:

$$
\begin{gathered}
H(\xi)=1+2 r_{+} A \xi, \\
E(\xi)=1+\left(r_{+}+r_{-}\right) A \xi, \\
J(\xi)=1+2 r_{+} A \xi+r_{+}\left(r_{+}-r_{-}\right) A^{2} \xi^{2} .
\end{gathered}
$$

The following cases are especially interesting.

(1) For $r_{+}=r_{-}$we have $H(y)=E(y)=J(y)=1$ $+2 r_{+} A y$. The nut is at $y=-1 /\left(2 r_{+} A\right)$.

(2) For $r_{+}=0$, we have $H(y)=J(y)=1, E(y)=1$ $+r_{-} A y$. The bubble is at $y=-1 /\left(r_{-} A\right)$.

(3) For $r_{-}=0, E(y)=1+r_{+} A y, J(y)=E(y)^{2}$. The bubble is at $y=-1 /\left(2 r_{+} A\right)$.

We can now rewrite the KK $C$ metrics with bubbles or GPS monopoles in a way that the bubbles or monopoles are located at precisely these same values of $y$. This requires that in the $C$ metrics we make coordinate changes of the form

$$
\begin{aligned}
& y^{-1} \rightarrow y^{-1}+r_{0} A, \\
& x^{-1} \rightarrow x^{-1}+r_{0} A,
\end{aligned}
$$

where by adequately choosing $r_{0}$ we can locate the bubbles where required.

After some straightforward manipulations, we can write all these metrics in the following unified form: 


$$
\begin{aligned}
d s^{2}= & \frac{H(y) E(y) J(x)}{H(x) E(x) J(y)}\left(d x^{5}+2 x \sqrt{r_{+} r_{-}} d \varphi\right)^{2} \\
& +\frac{1}{A^{2}(x-y)^{2}}[H(x) E(x) \\
& \times\left(K(y) d t^{2}-\frac{J(y)}{E(y) H(y) K(y)} d y^{2}\right) \\
& \left.+J(y)\left(\frac{d x^{2}}{K(x)}+\frac{K(x) E(x) H(x)}{J(x)} d \varphi^{2}\right)\right] .
\end{aligned}
$$

The functions $H, E, J$, are as given above. Despite its complicated aspect, this metric easily reproduces all the required limiting solutions if the function $K$ is properly chosen. Precisely, one must take the following.

(1) For $r_{+}=r_{-}$,

$$
K(\xi)=\frac{1}{H(\xi)}-\xi^{2}
$$

(2) For $r_{+}=0$,

$$
K(\xi)=E(\xi)^{2}-\xi^{2}
$$

(3) For $r_{-}=0$,

$$
K(\xi)=H(\xi)^{2}-\xi^{2}
$$

Whatever the $C$ metric for magnetic bubbles is, it must be possible to write it in a way that these solutions are found. Additionally, the static limit $A \rightarrow 0$ requires that, for large $y, K(y) \sim-y^{2}$, and for $x \sim 1, K(x) \sim 1-x^{2}$. Notice that there are two forms of a neutral bubble $C$ metric; they are related by a coordinate change such as in Eq. (B5). Alternative forms are possible, but the magnetic potential term gets more complicated. Unfortunately, we have not found any simple enough function that reproduces the above limits and, simultaneously, solves the five-dimensional Einstein equations.

If we tried to aim at finding only the most interesting case, the solution containing massless monopoles, the approach just described might not be adequate, and other simplifications may be more useful. For massless objects, the polynomial functions in the $C$ metric have to be even (or, more properly, one expects them to be even for at least some choice of coordinates). This property can easily be seen to be related to the no-external-force condition in the $(x, \varphi)$ part of the metric, or equivalently, the vanishing of the mass in the (static limit of the) $(t, y)$ part (see [28]). However, even in this particular case the solution has remained elusive.
[1] M. J. Duff, R. R. Khuri, and J. X. Lu, Phys. Rep. 259, 213 (1995).

[2] R. Gregory and R. Laflamme, Phys. Rev. Lett. 70, 2837 (1993).

[3] G. W. Gibbons, G. T. Horowitz, and P. K. Townsend, Class. Quantum Grav. 12, 297 (1995).

[4] E. Witten, Nucl. Phys. B195, 481 (1982).

[5] R. Sorkin, Phys. Rev. Lett. 51, 87 (1983).

[6] D. J. Gross and M. J. Perry, Nucl. Phys. B226, 29 (1983).

[7] G. W. Gibbons, Nucl. Phys. B207, 337 (1982).

[8] G. W. Gibbons, in Field Theory, Quantum Gravity and Strings, edited by H. De Vega and N. Sánchez (Springer, New York, 1986).

[9] C. Vafa, Nucl. Phys. B469, 403 (1996).

[10] S. Adams and G. Stuck, “'The Isometry Group of a Compact Lorentz Manifold,' UMD Math report, 1995 (unpublished).

[11] F. J. Ynduráin, Phys. Lett. B 256, 15 (1991).

[12] F. J. Dyson, Phys. Rev. 85, 631 (1952).

[13] G. W. Gibbons and D. Rasheed, 'Dyson-pairs and zero-mass black holes,' Report No. hep-th/9604177 (unpublished).

[14] P. Dobiasch and D. Maison, Gen. Relativ. Gravit. 14, 231 (1982).

[15] G. W. Gibbons and D. L. Wiltshire, Ann. Phys. (N.Y.) 167, 201 (1986).

[16] M. Cvetič and D. Youm, Phys. Rev. Lett. 75, 4165 (1995).

[17] D. N. Page, Phys. Lett. 78B, 249 (1978).
[18] A. Achúcarro, R. Gregory, and K. Kuijken, Phys. Rev. D 52, 5729 (1995).

[19] R. D. Young, Phys. Rev. D 28, 2420 (1983).

[20] S. W. Hawking and G. F. R. Ellis, The Large Scale Structure of the Universe (Cambridge University Press, Cambridge, England, 1973).

[21] D. Eardley, D. Kastor, J. Traschen, and G. Horowitz, Phys. Rev. Lett. 75, 3390 (1995).

[22] F. Dowker, J. Gauntlett, D. Kastor, and J. Traschen, Phys. Rev. D 49, 2909 (1994).

[23] F. Dowker, J. Gauntlett, S. Giddings, and G. Horowitz, Phys. Rev. D 50, 2662 (1994).

[24] S. W. Hawking and S. F. Ross, Phys. Rev. Lett. 75, 3382 (1995).

[25] P. Ginsparg and M. J. Perry, Nucl. Phys. B222, 245 (1983).

[26] S. J. Poletti and D. L. Wiltshire, Phys. Rev. D 50, 7260 (1994).

[27] S. W. Hawking, G. T. Horowitz, and S. F. Ross, Phys. Rev. D 51, 4302 (1995).

[28] R. Emparan, Phys. Lett. B 387, 721 (1996).

[29] S. W. Hawking, presented at DAMTP seminar (unpublished).

[30] R. Emparan, Phys. Rev. Lett. 75, 3386 (1995).

[31] F. Dowker, J. P. Gauntlett, G. W. Gibbons, and G. T. Horowitz, Phys. Rev. D 52, 6929 (1995).

[32] D. Rasheed, Nucl. Phys. B454, 379 (1995).

[33] K. Behrndt, Nucl. Phys. B455, 188 (1995); R. Kallosh and A. Linde, Phys. Rev. D 52, 7137 (1995). 